\title{
Does Intraoperative Diuretic Therapy Affect the Thoracic Fluid Content and Clinical Outcome in Patients Undergoing Mitral Valve Surgery?
}

\author{
Minati Choudhury ${ }^{1} \quad$ Jitin Narula ${ }^{1} \quad$ Kulbhushan Saini $^{1} \quad$ Poonam Malhotra Kapoor $^{1} \quad$ Usha Kiran $^{1}$ \\ ${ }^{1}$ Cardiothoracic Sciences Centre, All India Institute of Medical \\ Address for correspondence Minati Choudhury, MD, PG Dip, \\ Cardiothoracic Sciences Centre, All India Institute of Medical \\ Sciences, Room No 10, 7th floor, Ansari Nagar West, \\ New Delhi, 110029, India (e-mail: minati.2002@gmail.com).
}

J Card Crit Care:2020;4:86-90

\begin{abstract}
Pulmonary congestion is inevitable in valvular heart disease. The condition worsens when a patient undergoes cardiopulmonary bypass (CPB). Thoracic fluid content (TFC) is an indicator of total volume status of lung in health and disease. We hypothesize that intraoperative diuretic therapy can improve the hemodynamic and clinical outcome in patients undergoing mitral valve surgery by decreasing TFC as measured by impedance cardiography (ICG).

Sixty adult patients with critical mitral stenosis scheduled for mitral valve surgery divided into diuretic ( $\mathrm{Gr} D, n=30$ ) and control $(\mathrm{Gr} C, n=30$ ) group. One $\mathrm{mg} / \mathrm{kg}$ of furosemide was administered before CPB to $\mathrm{Gr} D$ and similar volume of normal saline to $\mathrm{Gr}$ C. Hemodynamic and TFC measurements were done by index of contractility (ICON), NICOM monitor at baseline, before anesthesia induction (T1), post skin closure (T2), postoperatively at 6th hour (T3), 24th hour (T4), and 48th hour (T5). The duration of mechanical ventilation (hour), intensive care unit stay (day) and inotropic score was significantly higher in $\operatorname{Gr} C(5.29 \pm 1.4$ vs. $2.15 \pm 1.1 ; p=0.001 ; 2.11 \pm 0.64$ vs. $1.67 \pm$ $0.57, p=0.002 ; 9 \pm 5.4$ vs. $5.8 \pm 3.2, p=0.05)$, respectively. Three patients in $\operatorname{Gr} C$ developed respiratory complication during their course of hospital stay. The baseline TFC value was comparable $(p=0.08)$. In $\operatorname{Gr} C$. it gradually increased over time and never reached the baseline value, whereas in Gr D, there was slight increase in TFC till 6th postoperative hour and it came below the baseline value at 48th hour. A significantly higher TFC value in $\mathrm{Gr}$ C in comparison with Gr D from 6th to 48th postoperative hour ( $p=0.005,0.000$, and 0.005 , respectively) was observed.

\section{Keywords}

- thoracic fluid content

- diuretic therapy

- clinical outcome

The ICON had a gradual improvement from 12th over 48th postoperative hour in $\mathrm{Gr} \mathrm{D}$ in comparison to $\mathrm{Gr}$ C. The systemic vascular resistance index was decreased over time in $\mathrm{Gr}$ C, whereas in Gr D there was a mild fall at the end of surgery and it came back to near the baseline value at 48th postoperative hour. A continuous decrease in $\mathrm{DO}_{2} \mathrm{I}$ except at 6 th postoperative hour was seen in $\mathrm{Gr} C$, whereas it remained near the baseline value in $\mathrm{Gr} D$.
\end{abstract}

published online

November 6, 2020
DOI https://doi.org/

10.1055/s-0040-1721186 ISSN 2457-0206. (c) 2020. Official Publication of The Simulation Society (TSS), accredited by International Society of Cardiovascular Ultrasound (ISCU).

This is an open access article published by Thieme under the terms of the Creative Commons Attribution-NonDerivative-NonCommercial-License, permitting copying and reproduction so long as the original work is given appropriate credit. Contents may not be used for commercial purposes, or adapted, remixed, transformed or built upon. (https://creativecommons.org/licenses/by-nc-nd/4.0/) Thieme Medical and Scientific Publishers Pvt. Ltd., A-12, 2nd Floor, Sector 2, Noida-201301 UP, India 
Linear regression analysis showed significant direct correlation of TFC with intraoperative fluid balance $(r=0.524, p=0.001)$, cumulative fluid balance $(r=0.680, p=0.000)$ and both peak and mean airway pressure ( $r=0.436, p=0.001$ and $r=0.548, p=0.001$, respectively). We concluded that TFC is clearly influenced by intraoperative diuretic therapy. A decrease in TFC has an association with better hemodynamic parameters that could find interesting clinical applications in the decision, whether or not to include a diuretic as a routine therapy during intraoperative management in valve surgery patients.

\section{Introduction}

Long-standing valvular heart disease may result in an increase in left atrial pressure (LAP) and pulmonary congestion. This subsequently leads to an increase in extravascular lung water (EVLW). ${ }^{1}$ Cardiopulmonary bypass (CPB) generates cytokines and other inflammatory mediators that subsequently lead to increase in lung water and deranged pulmonary function during the postoperative period. ${ }^{2}$ Lung water also increased by anesthesia, surgery, muscle paralysis, and surgical trauma.,4 Wellnlofer et al in one of their study concluded that percutaneous mitral valve commissurotomy leads to symptomatic improvement in mitral stenosis by decrease in lung water without any change in cardiac output or LAP. ${ }^{1}$ Clinical assessment of cardiac status is incomplete without an assessment of volume status of especially that of lung. Thoracic fluid content (TFC) is an indicator of total fluid volume (both intracellular and extracellular). Sanidas et al in one of their reports shown that both hypertensive men and women who were treated especially with diuretics showed a significantly lower TFC in comparison with patients treated with other antihypertensive drugs, untreated patients, and healthy $2 .^{5}$ The probable explanation may be a decrease in right ventricular strain following diuretic treatment that leads to a decrease in back pressure in lung vessels. Impedance cardiography (ICG) permits dynamic and noninvasive monitoring of TFC and hemodynamic in hospital setting. ${ }^{6}$ Keeping all these facts in mind we aim to (1) investigate the potential effect of intraoperative diuretic therapy on TFC using ICG, (2) to examine whether empiric administration of diuretic has an effect on hemodynamic and clinical outcome in patients undergoing mitral valve surgery.

\section{Methods}

This randomized, prospective, controlled, double-blind study was undertaken in a tertiary health care center, teaching and research institute between the periods of January 2012 and December 2016. Sixty adult patients with critical mitral valve disease, in New York Heart Association 3/4 scheduled for mitral valve surgery, were enrolled in the study after institutional ethics committee approval and written informed consent from the patients or guardian. The patients with severe ventricular dysfunction, preoperative inotropic support/ mechanical ventilation, redo surgery, renal/hepatic insufficiency, uncontrolled hypertension, recurrent arrhythmias, and dermatological problem (interfere with noninvasive cardiac output monitor (NICOM) patch placement) were excluded from the study. Patients with postoperative complication requiring return to operating room were excluded from the final analysis.

The anesthesia and CPB management were similar for all patients and according to the routine protocol. None of the patients received any diuretics or angiotensin-converting enzyme inhibitor prior to surgery. Standard monitoring for any other cardiac patients was applied in addition to NICOM monitor. Four dual sensors were placed on either side of neck and thorax for bioimpedance CO monitoring. Randomization was done with computer-generated sealed envelope technique following anesthesia induction. The patients were divided into two groups: diuretic group ( $\mathrm{Gr} \mathrm{D}, n=30$ ) and control group ( $\mathrm{Gr} \mathrm{C}, n=30$ ). The $\mathrm{Gr} D$ patients received $1 \mathrm{mg} /$ $\mathrm{kg}$ of furosemide before going on CPB and $\mathrm{Gr} C$ received an equal volume of placebo. Hemodynamic and TFC measurements were done by index of contractility (ICON), NICOM monitor (Osypka Medical, Inc., La Jolla, California, United States) at predetermined set points (baseline, before anesthesia induction [T1], post skin closure [T2], postoperatively at 6th hour [T3], 24th hour [T4], and 48th hour [T5]. During CPB, the patient was cooled down to 32 to $34^{\circ} \mathrm{C}$ and hematocrit was maintained between 25 and $28^{\circ} \mathrm{C}$. The clinical outcome of all the patients was measured during the period of hospital stay.

\section{Statistical Analysis}

Software, Sigma stat version 11.5 (SPSS, Inc., Chicago, Illinois, United States), was used for the analysis. The continuous variables were expressed in mean \pm standard deviation/ median (range). The mean difference in the continuous variables at different time points was measured by Student's $t$-test or stage 2 analysis of variance. To study the correlation between variables, we used linear regression analysis. For all the measurements, a $p$-value of $<0.05$ was considered to be significant.

\section{Results}

The final analysis included 58 subjects in total, with 30 patients in Gr D and 28 patients in Gr C. Two patients from $\mathrm{Gr} \mathrm{C}$ were excluded from final analysis because of mediastinal bleeding and re-exploration. The demographic 
and preoperative variables are comparable in both the groups (-Table 1). The CPB and AoX $\mathrm{Cl}$ duration, duration of hospital stay, baseline peak, and mean airway pressure were similar in both the groups (-Table 2). However, the duration of mechanical ventilation (hour), intensive care unit (ICU) stay (day), and inotropic score was significantly

Table 1 Demographics, preoperative variables

\begin{tabular}{|l|l|l|l|}
\hline Variables & Gr C, $\boldsymbol{n}=\mathbf{3 0}$ & Gr D, $\boldsymbol{n}=\mathbf{2 8}$ & $\boldsymbol{p}$-Value \\
\hline Age $(\mathrm{y})$ & $32.7 \pm 6.9$ & $31.64 \pm 6.6$ & 0.71 \\
\hline Sex $(\mathrm{M} / \mathrm{F})$ & $11 / 14$ & $10 / 15$ & 0.08 \\
\hline Height $(\mathrm{cm})$ & $153.6 \pm 7.1$ & $158.7 \pm 9.7$ & 0.11 \\
\hline Weight $(\mathrm{kg})$ & $47.76 \pm 12.6$ & $47.36 \pm 9.4$ & 0.23 \\
\hline Hct $(\%)$ & $38.6 \pm 4.8$ & $39.5 \pm 2.8$ & 0.10 \\
\hline BSA (m²) & $1.41 \pm 0.18$ & $1.45 \pm 0.18$ & 0.9 \\
\hline Beta-blocker (\%) & $7(28)$ & $9(36)$ & 0.54 \\
\hline Digoxin (\%) & $12(48)$ & $10(40)$ & 0.56 \\
\hline Diuretics (\%) & $16(64)$ & $14(56)$ & 0.56 \\
\hline ACEl (\%) & $3(12)$ & $0(0)$ & 0.07 \\
\hline Ejection fraction & $49 \pm 2$ & $48.4 \pm 3$ & 0.36 \\
\hline PAH (n); Mod, Sev & 12,13 & 11,14 & 0.12 \\
\hline
\end{tabular}

Abbreviations: ACEI, angiotensin-converting enzyme; BSA, body surface area; Hct, hematocrit; PAH, pulmonary hypertension.

Table 2 Durations and clinical outcome parameters

\begin{tabular}{|l|l|l|l|}
\hline Variables & Gr C, $\boldsymbol{n}=\mathbf{3 0}$ & Gr D, $\boldsymbol{n}=\mathbf{2 8}$ & $\boldsymbol{p}$-Value \\
\hline CPB dur (min) & $84.64 \pm 27.35$ & $81.1 \pm 21.1$ & 0.24 \\
\hline AoX cl (min) & $58.1 \pm 21.1$ & $61.9 \pm 27.0$ & 0.17 \\
\hline Mech vent(h) & $5.29 \pm 1.4$ & $2.15 \pm 1.1^{*}$ & $0001^{*}$ \\
\hline ICU stay (h) & $2.11 \pm 0.64$ & $1.67 \pm 0.57^{*}$ & $0.002^{*}$ \\
\hline Hospital stay (d) & $6 \pm 5.4$ & $5.8 \pm 3.2$ & 0.32 \\
\hline Inotrope score & $9 \pm 5.4$ & $5.8 \pm 3.2$ & $0.05^{*}$ \\
\hline $\begin{array}{l}\text { Peak AWP } \\
\text { T1 } \\
\text { T5 }\end{array}$ & $18.7 \pm 4.5$ & $18.8 \pm 3.6$ & 0.32 \\
\hline $\begin{array}{l}\text { Mean AWP } \\
\text { T1 } \\
\text { T5 }\end{array}$ & $19.6 \pm 4.6, p=0.8$ & $\begin{array}{l}16.2 \pm 2.4^{*} \\
p=0.05\end{array}$ & $0.001^{*}$ \\
\hline Bleeding & $13.5 \pm 3.7, p=0.2$ & $13.5 \pm 3.2$ & 0.52 \\
\hline Resp complication & 3 & $p=0.23$ & 0.43 \\
\hline
\end{tabular}

*0.0001

Abbreviations: AWP, airway pressure; CPB, cardiopulmonary bypass; ICU, intensive care unit. higher in Gr C (5.29 \pm 1.4 vs. $2.15 \pm 1.1 ; p=0.001 ; 2.11 \pm$ 0.64 vs. $1.67 \pm 0.57,0.002 ; 9 \pm 5.4$ vs. $5.8 \pm 3.2, p=0.05$ ), respectively. Three patients in $\mathrm{Gr} C$ developed respiratory complication during their course of hospital stay, whereas none from Gr D faced the similar complication. Though the peak airway pressure in $\mathrm{Gr} C$ at $\mathrm{T} 5$ was statistically higher than Gr D, it was not clinically significant (-Table 2). While studying the TFC, the authors noted that TFC value in both the groups was comparable in both the groups at baseline $(p=0.08)$. In $\mathrm{Gr} C$, it gradually increased over time, there was slight decrease at 48th hour, and it never reached the baseline value (-Table 3 ). However, in Gr D, there was slight increase in TFC till 6th postoperative hour and it came below the baseline value at 48th hour postoperatively ( - Table 3 ). Intergroup comparison revealed a significantly higher TFC value in $\mathrm{Gr} C$ in comparison with Gr D from 6th to 48th postoperative hour ( $p=0.005,0.000$, and 0.005 , respectively).

While analyzing the hemodynamic parameters ( - Table 4), the authors noticed that the ICON is similar in both the groups at baseline, and then a gradual improvement from 12th over 48th postoperative hour in $\mathrm{Gr} \mathrm{D}$ in comparison to $\mathrm{Gr} \mathrm{C}$. The systemic vascular resistance index was decreased over time in Gr C, whereas in Gr D there was a mild fall at the end of surgery and it came back to near the baseline value at 48th postoperative hour. The change in $\mathrm{CaO}_{2}$ over time was comparable in both the groups. A minimal and comparable decrease in trend from baseline was observed in both the groups post-surgery till 24th postoperative hour. The baseline $\mathrm{CI}$ was comparable in both the groups and it was improved over time in both the groups, which was statistically significant. A continuous decrease in $\mathrm{DO}_{2} \mathrm{I}$ except at 6th postoperative hour was seen in $\mathrm{Gr} C$, whereas it remains near the baseline value in Gr D. Surprisingly, the stroke volume variation was more in $\mathrm{Gr} \mathrm{C}$ in comparison to $\mathrm{Gr} \mathrm{D}$. However, these values are not clinically relevant. The baseline right atrial pressure (RAP) was higher than normal in both the groups, remained near normal post-surgery, and thereafter Gr D did not show a much decrease in RAP in comparison to $\mathrm{Gr}$ C.

Linear regression analysis was done to compare TFC with the fluid balance and other variables (-Table 5). We observed a significant direct correlation of TFC with intraoperative fluid balance $(r=0.524, p=0.001)$, cumulative fluid balance $(r=0.680, p=0.000)$, and both peak and mean airway pressure $(r=0.436, p=0.001$ and $r=0.548, p=0.001$, respectively).

Table 3 Thoracic fluid content (TFC) values at different time points

\begin{tabular}{|l|l|l|l|}
\hline Time & Gr C, $\boldsymbol{n}=25$ & Gr D, $\boldsymbol{n}=\mathbf{2 5}$ & -Value \\
\hline Baseline(T1) & $25.4 \pm 7.3^{*}$ & $24.8 \pm 3.1$ & 0.08 \\
\hline Post skin closure (T2) & $26.3 \pm 7.1^{*}$ & $26.4 \pm 6.1$ & 0.06 \\
\hline 6 hours postoperatively (T3) & $29.8 \pm 7.4^{*}$ & $26.8 \pm 5.9^{*}$ & 0.005 \\
\hline 24 hours postoperatively (T4) & $30.2 \pm 6.8^{*}$ & $22.8 \pm .3^{*}$ & 0.000 \\
\hline 48 hours postoperatively (T5) & $28 \pm 6.2^{*}$ & $21.22 \pm 1.2^{*}$ & 0.005 \\
\hline
\end{tabular}

${ }^{*} 0.0001$ 
Table 4 Hemodynamic parameters (values expressed in mean \pm SD or median)

\begin{tabular}{|c|c|c|c|c|c|}
\hline Variable & $\mathrm{T} 1$ & $\mathrm{~T} 2$ & T3 & T4 & T5 \\
\hline \multicolumn{6}{|l|}{ ICON } \\
\hline GrC & $51.2 \pm 12.2$ & $58.7 \pm 23.5$ & $51.5 \pm 14.9$ & $52.5 \pm 14.6$ & $58.8 \pm 10.3$ \\
\hline Gr D & $51.6 \pm 5.9$ & $54.5 \pm 19.7$ & $51.7 \pm 19.5^{*}$ & $61.3 \pm 18.2^{*}$ & $63.3 \pm 17^{*}$ \\
\hline \multicolumn{6}{|l|}{ SVRI } \\
\hline GrC & 2399 & $1505^{*}$ & 1529* & $1766^{*}$ & $1616^{*}$ \\
\hline GrD & 2440 & $1690^{*}$ & $1699^{*}$ & $1967^{*}$ & $2112^{*}$ \\
\hline \multicolumn{6}{|l|}{$\mathrm{CaO}_{2}$} \\
\hline GrC & $18.4 \pm 2.2$ & $13.3 \pm 3.2^{*}$ & $14.2 \pm 1.2^{*}$ & $15.9 \pm 0.7$ & $17.2 \pm 0.8^{*}$ \\
\hline Gr D & $18.9 \pm 2.3$ & $13.8 \pm 2.5^{*}$ & $15.5 \pm 1.3^{*}$ & $16.3 \pm 1.7^{*}$ & $17.5 \pm 1.1^{*}$ \\
\hline \multicolumn{6}{|l|}{$\mathrm{DO}_{2} \mathrm{I}$} \\
\hline GrC & 521 & $456^{*}$ & $563^{*}$ & $470^{*}$ & 409 \\
\hline GrD & 539 & $578^{*}$ & 500 & $568^{*}$ & 534 \\
\hline \multicolumn{6}{|l|}{$\mathrm{Cl}$} \\
\hline GrC & $2.6 \pm 1.1$ & $3.8 \pm 1.1^{*}$ & $3.7 \pm 0.91^{*}$ & $3.7 \pm 0.89^{*}$ & $3.8 \pm 0.82^{*}$ \\
\hline Gr D & $2.6 \pm 0.63$ & $3.4 \pm 0.93^{*}$ & $3.5 \pm 0.80^{*}$ & $3.7 \pm 0.96^{*}$ & $3.9 \pm 0.73^{*}$ \\
\hline \multicolumn{6}{|l|}{ SVV } \\
\hline GrC & $13.2 \pm 6.1$ & $16.7 \pm 5.7^{*}$ & $12.7 \pm 3.3$ & $9.8 \pm 1.9^{*}$ & $9.8 \pm 1.3^{*}$ \\
\hline GrD & $14.3 \pm 8.6$ & $14.04 \pm 6.4$ & $11.48 \pm 3.5$ & $10.2 \pm 2.0^{*}$ & $9.8 \pm 1.2^{*}$ \\
\hline \multicolumn{6}{|l|}{ RAP } \\
\hline $\mathrm{GrC}$ & $17.8 \pm 2.7$ & $7.9 \pm 2.6^{*}$ & $8.7 \pm 1.5^{*}$ & $9.7 \pm 1.6^{*}$ & $9.8 \pm 1.3^{*}$ \\
\hline GrD & $17.2 \pm 3.6$ & $7.6 \pm 2.8^{*}$ & $8.6 \pm 1.8^{*}$ & $9.2 \pm 1.4^{*}$ & $9.8 \pm 1.2^{*}$ \\
\hline
\end{tabular}

*0.0001

Abbreviations: ICON, index of contractility; RAP, right arterial pressure; SD, standard deviation; SVRI, systemic vascular resistance index; SVV, stroke volume variation.

Table 5 Linear regression comparing TFC with other parameters

\begin{tabular}{|l|l|l|}
\hline Variables & $\begin{array}{l}\text { Correlation } \\
\text { Coefficient }\end{array}$ & $p$-Value \\
\hline Intraoperative fluid balance & 0.524 & $0.001^{*}$ \\
\hline Cumulative fluid balance & 0.680 & $0.000^{*}$ \\
\hline CPB time & 0.316 & 0.025 \\
\hline Hours of mechanical ventilation & 0.585 & $0.000^{*}$ \\
\hline Duration of ICU stay & 0.212 & 0.139 \\
\hline Duration of inotrope & 0.243 & 0.089 \\
\hline$p$ peak & 0.436 & $0.001^{*}$ \\
\hline$p$ mean & 0.548 & $0.001^{*}$ \\
\hline
\end{tabular}

${ }^{*} 0.0001$

Abbreviations: CPB, cardiopulmonary bypass; ICU, intensive care unit; TFC, thoracic fluid content.

\section{Discussion}

Assessment of fluid volume status of lung is essential for complete clinical assessment of cardiac status. TFC is measured by reading the impedance to electrical conductivity through chest wall. This qualitative measure is useful to track changes in thoracic fluid levels over time. Kossari et al assessed the treatment options in their hemodialysis patients using TFC where fluid balance is critical. ${ }^{7}$ Engineer et al in their study demonstrated the utility of TFC along with $\mathrm{Cl}$ to an orthostatic challenge that helps in differential diagnosis of heart failure versus chronic obstructive pulmonary disease patients presenting in emergency department for shortness of breath. It may also be useful to track the response to treatment of volume overload in heart failure patients and treatment of acute respiratory distress syndrome or acute lung injury where thoracic fluids are often abnormally high. ${ }^{8}$

Significant pulmonary complications occur in some cardiac surgical cases due to increase in lung water and resultant atelectasis following CPB. Ultrafiltration has been shown to decrease total body water and postoperative blood loss and improve the alveolar to arterial oxygen gradient and pulmonary compliance. ${ }^{9}$ Ultrafiltration is used by many centers to remove lung water and improve lung recruitment during the postoperative period. This is, however, is at times not cost-effective for some patients and controversial results in some. ${ }^{10}$

Diuretic therapy is a routine part of management of patients with congestive cardiac failure. This is cheap and easily available. Longer duration of mechanical ventilation and ICU stay and an increase in TFC value in Gr C patients clearly indicate the presence of lung congestion as an important cause of weaning failure in these patients. This finding of ours is supported by previous reports. ${ }^{11}$ The improved breathing efforts, less duration of mechanical ventilation, and less inotropic score in Gr D are also supported by previous 
reports in which TFC was able to follow up the hemodynamic effects of diuretic in hypertensive patients. ${ }^{5}$ The utility of intraoperative diuretics and monitoring its effect by TFC can be used as a low-cost therapeutic and diagnostic method for detection and treatment of impending weaning failure in high risk mitral valve disease cases. The impact of lung congestion in our Gr C patients was represented by increased TFC. This explanation is supported by some other findings who in their randomized controlled trial reported that brain natriuretic peptide guided protocol for weaning showed better patient outcome compared with traditional weaning protocol..$^{12}$ TFC showed good correlation with fluid volume during cardiac surgery. ${ }^{13}$ Premature failed weaning leads to prolonged intubation, subsequent alteration of cardiovascular parameters, and increases morbidity and mortality. ${ }^{14}$ Similarly prolong mechanical ventilation is also associated with serious complications..$^{15}$ There is ongoing meticulous research to know the best predictor of delayed weaning in heart valve surgery, its estimation methods, and therapeutic/preventive options. ${ }^{16,17}$

Ventricular dysfunction and increased lung water are the common predictors of prolong mechanical ventilation and increase inotrope use, whereas echocardiography is the frequently used method to estimate cardiac function. TFC by ICON monitor is a simple and noninvasive method to estimate the serial changes in lung water without any need for operator experience. This is easily interrelated without any interobserver variability because it is presented in numerical values that have minimal fluctuation and it does not involve any ongoing cost. Poor pulmonary function in $\mathrm{Gr} \mathrm{C}$ with high TFC value can be taken as an alarming sign in cardiac surgical patients to initiate diuretic therapy before weaning from mechanical ventilation.

\section{Limitation of the Study}

1. Single-center study.

2. We had not taken any patient who undergoes ultrafiltration for a comparison. We suggested that our findings need to be confirmed in future studies in another patient population.

3. We did not measure cardiac markers such as brain natriuretic peptide.

\section{Conclusion}

TFC is clearly influenced by intraoperative diuretic therapy. A decrease in TFC has an association with better hemodynamic parameters that could find interesting clinical applications in the decision, whether or not to include a diuretic as a routine therapy during intraoperative management in valve surgery patients.

\section{Declaration}

No conflict of interest.

\section{References}

1 Wellnhofer E, Cramer C, Dreysse S, Fleck E. Lung water, hemodynamics and dyspnea before and after valvuloplasty in mitral stenosis. Int J Cardiol 2000;75(2-3):217-225

2 Heijmans JH, Liem KS, Damoiseaux GM, Maessen JG, Roekaerts PM. Pulmonary function and inflammatory markers in patients undergoing coronary revascularisation with or without cardiopulmonary bypass. Anaesthesia 2007;62(12):1233-1240

3 Apostolakis E, Filos KS, Koletsis E, Dougenis D. Lung dysfunction following cardiopulmonary bypass. J Card Surg 2010; 25(1):47-55

4 Babik B, Asztalos T, Peták F, DeákZI, Hantos Z. Changes in respiratorymechanicsduring cardiacsurgery.AnesthAnalg2003;96(5): 1280-1287

5 Sanidas EA, Grammatikopoulos K, Anastasiadis G, Papadopoulos D, Daskalaki M, Votteas V. Thoracic fluid content and impedance cardiography: a novel and promising noninvasive method for assessing the hemodynamic effects of diuretics in hypertensive patients. Hellenic J Cardiol 2009;50(6):465-471

6 van de Water JM, Mount BE, Chandra KM, Mitchell BP, Woodruff TA, Dalton ML. TFC (thoracic fluid content): a new parameter for assessment of changes in chest fluid volume. Am Surg 2005;71(1):81-86

7 Kossari N, Hufnagel G, Squara P. Bioreactance: a new tool for cardiac output and thoracic fluid content monitoring during hemodialysis. Hemodial Int 2009;13(4):512-517

8 Engineer RS, Benoit JL, Hicks CW, Kolattukudy SJ, Burkhoff D, Peacock WF. Hemodynamic changes as a diagnostic tool in acute heart failure-a pilot study. Am J Emerg Med 2012; 30(1):174-180

9 Williams GD, Ramamoorthy C, Chu L, et al. Modified and conventional ultrafiltration during pediatric cardiac surgery: clinical outcomes compared. J Thorac Cardiovasc Surg 2006; 132(6):1291-1298

10 Elayashy M, Madkour MA, Mahmoud AA, et al. Effect of ultrafiltration on extravascular lung water assessed by lung ultrasound in children undergoing cardiac surgery: a randomized prospective study. BMC Anesthesiol 2019;19(1):93

11 Folan L, Funk M. Measurement of thoracic fluid content in heart failure: the role of impedance cardiography. AACN Adv Crit Care 2008;19(1):47-55

12 Mekontso Dessap A, Roche-Campo F, Kouatchet A, et al. Natriuretic peptide-driven fluid management during ventilator weaning: a randomized controlled trial. Am J Respir Crit Care Med 2012;186(12):1256-1263

13 Perko MJ,Jarnvig IL, Højgaard-Rasmussen N, Eliasen K, Arendrup $\mathrm{H}$. Electric impedance for evaluation of body fluid balance in cardiac surgical patients. J Cardiothorac Vasc Anesth 2001; 15(1):44-48

14 Epstein SK, Ciubotaru RL, Wong JB. Effect of failed extubation on the outcome of mechanical ventilation. Chest 1997;112(1): 186-192

15 Cook DJ, Walter SD, Cook RJ, et al. Incidence of and risk factors for ventilator-associated pneumonia in critically ill patients. Ann Intern Med 1998;129(6):433-440

16 Papanikolaou J, Makris D, Saranteas T, et al. New insights into weaning from mechanical ventilation: left ventricular diastolic dysfunction is a key player. Intensive Care Med 2011; 37(12):1976-1985

17 de Meirelles Almeida CA, Nedel WL, Morais VD, Boniatti MM, de Almeida-Filho OC. Diastolic dysfunction as a predictor of weaning failure: a systematic review and meta-analysis. J Crit Care 2016;34:135-141 